\title{
Amphipathic Pentiptycene-Based Water-Resistant Cu-MOF for Efficient Oil/Water Separation
}

Minghui Zhang ${ }^{\dagger, \ddagger}$, Bingbing Guo ${ }^{\ddagger}$, Yang Feng ${ }^{\ddagger}$, Changsong Xie $^{\ddagger}, \mathrm{Xu} \mathrm{Han}^{\ddagger}, \mathrm{Xia} \mathrm{Kong}^{\S}$, Ben

$\mathrm{Xu}^{\ddagger, *}$, Liangliang Zhang ${ }^{+, \neq,{ }^{*}}$

${ }^{+}$Xi'an Institute of Flexible Electronics, Northwestern Polytechnical University, Xi'an 710072, China

${ }^{\ddagger}$ Key Laboratory of Unconventional Oil \& Gas Development, Ministry of Education, School of Materials

Science and Engineering, China University of Petroleum (East China), Qingdao, Shandong 266580, China

$\S$ School of Chemistry and Environmental Engineering, Shandong University of Science and Technology,

Qingdao 266510, China

E-mail: iamllzhang@nwpu.edu.cn; benxu@ upc.edu.cn;

\section{Supporting Information}

1. General procedures 1

2. Synthesis of ligand $\mathrm{H}_{2} \mathrm{~L}_{1}$

3. Synthesis of UPC-29 4

4. Single-crystal-to-single-crystal transformation 4

5. Crystal structure determination 4

6. PXRD, TGA and flux 5

7. Crystal Data 9

8. Photographs and SEM images 13

9. Water sorption isotherms 14

10. Water contact angle for UPC-29 and other MOFs 15

11. Photographs of oil/water separation 17

12. References 19 
1. General procedures. All chemicals and solvents in the syntheses were available from commercial sources and used without further purification. The ligand $\mathrm{H}_{2} \mathrm{~L}_{1}$ was prepared in $70 \%$ yield which was characterized by ${ }^{1} \mathrm{HNMR}$ (as shown in Fig. S2). The powder XRD data were collected by using an X-Pert PRO MPD diffractometer with $\mathrm{Cu}-\mathrm{K} \alpha$ radiation. Thermo-gravimetric analysis (TGA) curves were carried out on a Mettler Toledo TGA instrument under a $\mathrm{N}_{2}$ atmosphere with the heating rate of $10^{\circ} \mathrm{C} \cdot \mathrm{min}^{-1}$. The static water contact angles were measured on a contact angle measuring system (JC2000C1, POWEREACH) using the sessile drop method. A droplet $(5-\mu \mathrm{L})$ of distilled water was deposited on the surface of the samples at ambient temperature. The average value was obtained from at least five measurements per sample.

Oil/water separation experiment: Typically, moderate amounts of crystal samples were packaged by gauze and fixed in the middle of the glass tube, and the water was dyed by acid red 18. To ensure the contact of the tested objects with the crystals, the device was placed slantways. 
2. Synthesis of ligand $\mathbf{H}_{2} \mathbf{L} 1$. A mixture of anthracene (17.8 g, $\left.0.1 \mathrm{~mol}\right)$, benzoquinone $(5.4 \mathrm{~g}, 0.05 \mathrm{~mol})$ and $75 \mathrm{~mL}$ mesitylene was loaded into a $250 \mathrm{~mL}$ round-bottomed flask and refluxed for $24 \mathrm{~h}$. After reaction finished, the solid was filtered and digested in $100 \mathrm{~mL}$ of hot xylene twice and filtered. The crude products were purified with dichloromethane through a short silica gel column. The obtained hydroquinones $(1.01 \mathrm{~g}, 2.18 \mathrm{mmol})$, methyl 4-(bromomethyl)benzoate (1.50g, $6.54 \mathrm{mmol})$, $\mathrm{KI}(0.193 \mathrm{~g}, 1.16 \mathrm{mmol})$ and $\mathrm{K}_{2} \mathrm{CO}_{3}(1.40 \mathrm{~g}, 10.1 \mathrm{mmol})$ were added to $150 \mathrm{~mL}$ acetone and heated at reflux for $24 \mathrm{~h}$. After cooling to room temperature, the solvent was removed by rotary evaporation and the resulting precipitation was dissolved in DCM and washed with water to remove any insoluble salts. The organic layer was dried with $\mathrm{MgSO}_{4}$ and filtered, and the volatile was dried on rotary evaporator. The product was purified with dichloromethane through a short silica gel column (80\% yield). ${ }^{1} \mathrm{H}$ NMR (400 MHz, $\left.\mathrm{CDCl}_{3}\right): \delta / \mathrm{ppm}$ : 4.01 (s, 6H), 5.02 (s, 4H), $5.42(\mathrm{~s}, 4 \mathrm{H}), 6.91(\mathrm{dd}, 8 \mathrm{H}), 7.22(\mathrm{dd}, 8 \mathrm{H}), 7.72(\mathrm{~d}, 4 \mathrm{H}), 8.22(\mathrm{~d}, 4 \mathrm{H})$.

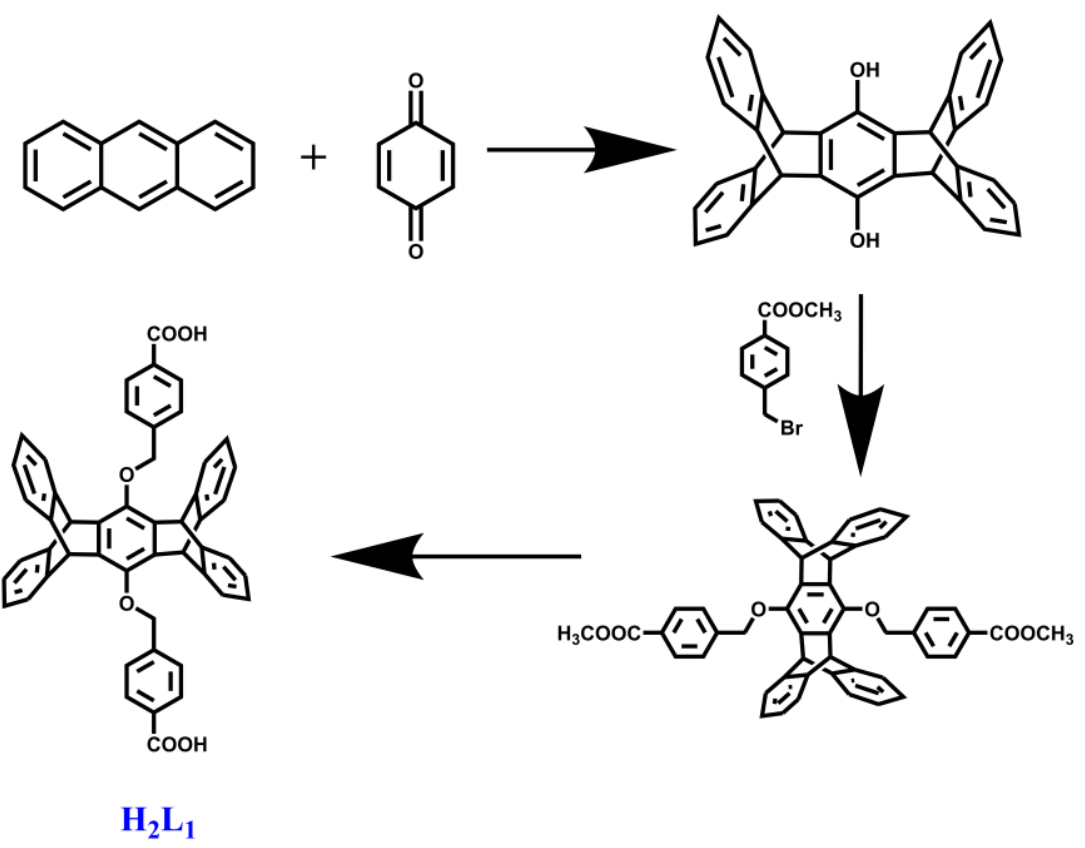

Scheme S1. Synthesis of $\mathrm{H}_{2} \mathrm{~L}_{1}$.

Then the ester was added in a mixture of THF $(75 \mathrm{~mL})$ and $2 \mathrm{M} \mathrm{NaOH}$ aqueous solution $(25 \mathrm{~mL})$. The mixture was stirred under reflux for 12 hours. After cooling to room temperature, the solvent was removed under a vacuum. Then dilute $\mathrm{HCl}$ was added to the residual aqueous solution until the solution was at $\mathrm{pH}=2$. The white precipitate was collected by filtration, washed with water, and dried to obtain $\mathrm{H}_{2} \mathbf{L}_{1}$

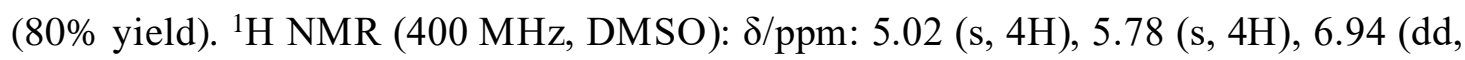


8H), $7.36(\mathrm{dd}, 8 \mathrm{H}), 7.88(\mathrm{~d}, 4 \mathrm{H}), 8.15(\mathrm{~d}, 4 \mathrm{H}), 13.08(\mathrm{~s}, 2 \mathrm{H})$.

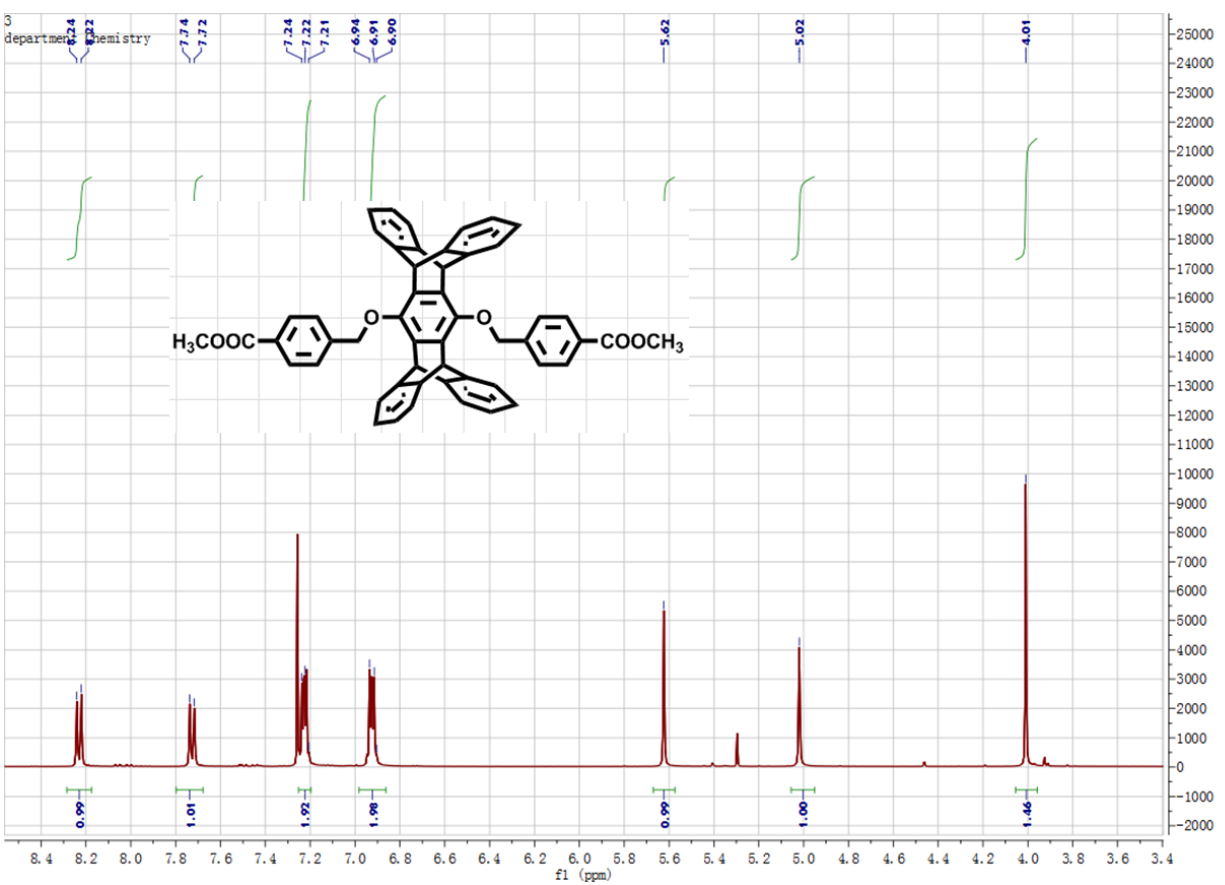

Figure S1. ${ }^{1} \mathrm{H}$ NMR spectrum of ester.

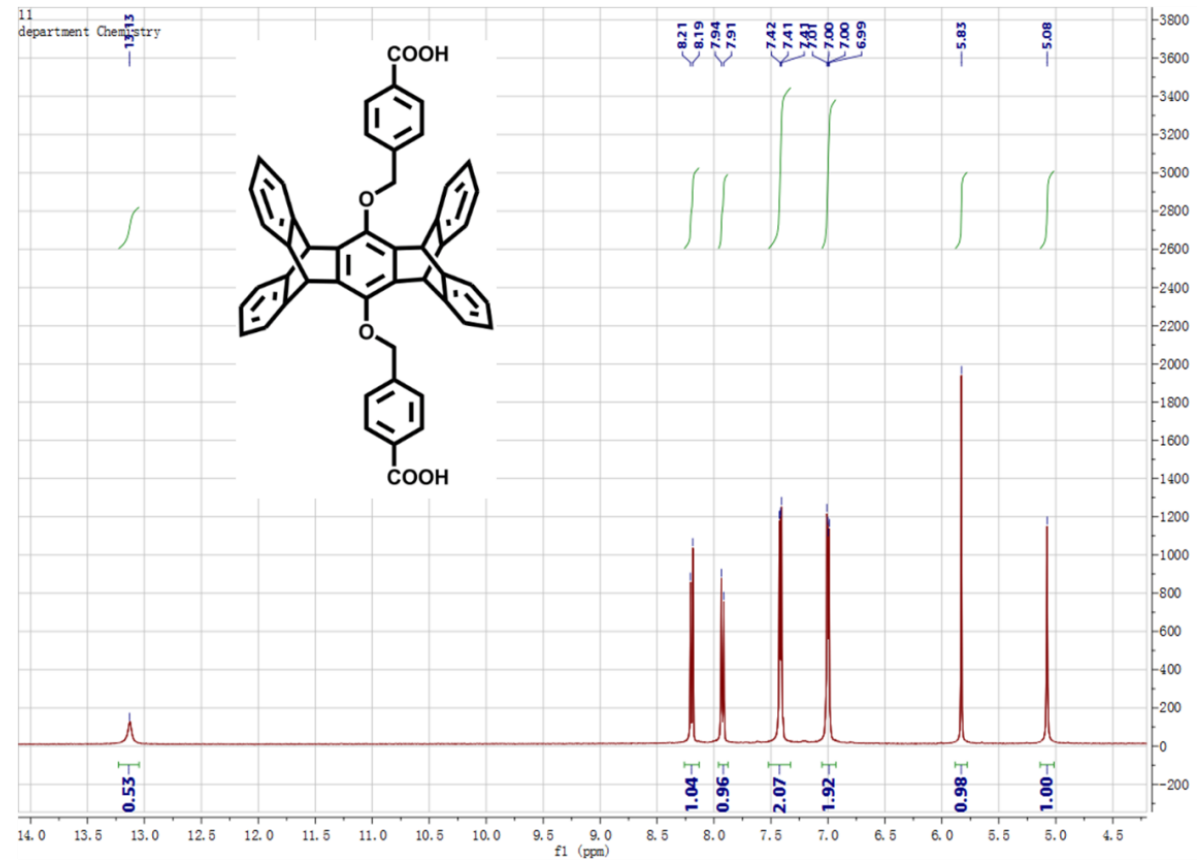

Figure S2. ${ }^{1} \mathrm{H}$ NMR spectrum of ligand $\mathrm{H}_{2} \mathrm{~L}_{1}$. 
3. Synthesis of UPC-29. $\mathrm{H}_{2} \mathrm{~L}_{1}(20 \mathrm{mg}, 0.027 \mathrm{mmol})$ and $\mathrm{Cu}\left(\mathrm{NO}_{3}\right)_{2} \cdot 3 \mathrm{H}_{2} \mathrm{O}(200$ $\mathrm{mg}, 1.066 \mathrm{mmol})$ were dissolved in DMF: EtOH: $\mathrm{H}_{2} \mathrm{O}(12 \mathrm{~mL}, \mathrm{~V}: \mathrm{V}: \mathrm{V}=5: 2: 1)$. The reaction solution was placed in an open glass via $(20 \mathrm{~mL})$. After 10 days later, green block crystals of UPC-29 were formed and collected. (yield: 65\%, based on ligand). Elemental analysis: calculated for $\left\{\left[\mathrm{Cu}_{2}\left(\mathrm{~L}_{1}{ }^{2-}\right)_{2}(\mathrm{DMF})_{2}\right] \cdot(\mathrm{DMF})_{5}\right\} \mathrm{n}$ $\left(\mathrm{C}_{121} \mathrm{H}_{113} \mathrm{Cu}_{2} \mathrm{~N}_{7} \mathrm{O}_{19}, \%\right)$ : C, 69.32; H, 5.44; N, 4.67; found: C, 70.53; H, 5.46; N, 4.62.

4. Single-crystal-to-single-crystal transformation In the solvent-induced process, the as-synthesized crystals of UPC-29 were soaked in water and bubbles were generated immediately on the crystals (as shown in Fig. S10). After 8 hrs later, the $\mathrm{H}_{2} \mathrm{O}$-induced crystals of UPC-29' were obtained and analyzed by using single crystal X-ray diffraction. Similar to the process of synthesis of UPC-29', crystals of UPC-29", were obtained after UPC-29 soaked in water overnight. Elemental analysis: calculated for $\quad$ UPC-29, $\quad\left\{\left[\mathrm{Cu}_{2}\left(\mathrm{~L}_{1}{ }^{2-}\right)_{2}(\mathrm{DMF})\left(\mathrm{H}_{2} \mathrm{O}\right)\right] \cdot(\mathrm{DMF})_{4} \bullet\left(\mathrm{H}_{2} \mathrm{O}\right){ }_{10}\right\} \mathrm{n}$ $\left(\mathrm{C}_{115} \mathrm{H}_{121} \mathrm{Cu}_{2} \mathrm{~N}_{5} \mathrm{O}_{28}, \%\right)$ : C, 64.29; H, 5.68; N, 3.26; found: C, 64.19; H, 5.82; N, 3.42. UPC-29" $\left\{\left[\mathrm{Cu}_{2}\left(\mathrm{~L}_{1}{ }^{2-}\right)_{2}\left(\mathrm{H}_{2} \mathrm{O}\right)_{2}\right] \bullet(\mathrm{DMF})\left(\mathrm{H}_{2} \mathrm{O}\right)_{6}\right\} \mathrm{n}\left(\mathrm{C}_{103} \mathrm{H}_{87} \mathrm{Cu}_{2} \mathrm{NO}_{21}, \%\right): \mathrm{C}, 68.66 ; \mathrm{H}$, 4.88; N, 0.7; found: C, 68.84; H, 4.61; N, 0.81 .

5. Crystal structure determination. Single crystal X-ray diffraction of UPC-29 were measured on an Agilent Xcalibur Eos Gemini diffractometer with $\mathrm{Cu} / \mathrm{K \alpha}$ radiation $(\lambda=1.54184 \AA)$ at $293 \mathrm{~K}$. All structures were solved by Olex 2 with the Superflip solution program and refined using the ShelXL refinement package with full-matrix least-squares on $F^{2}$. The SQUEEZE routine of PLATON was applied to remove the disordered solvent molecules, and then a new file was generated. The crystallographic data of UPC-29, UPC-29' and UPC-29', are given in Table S2, and selected bond lengths and angles are given in Table S3, S4 and S5. CCDC numbers for UPC-29, UPC-29' and UPC-29'” is 1569621, 1843019 and 1843020, respectively. 
6. PXRD, TGA and flux

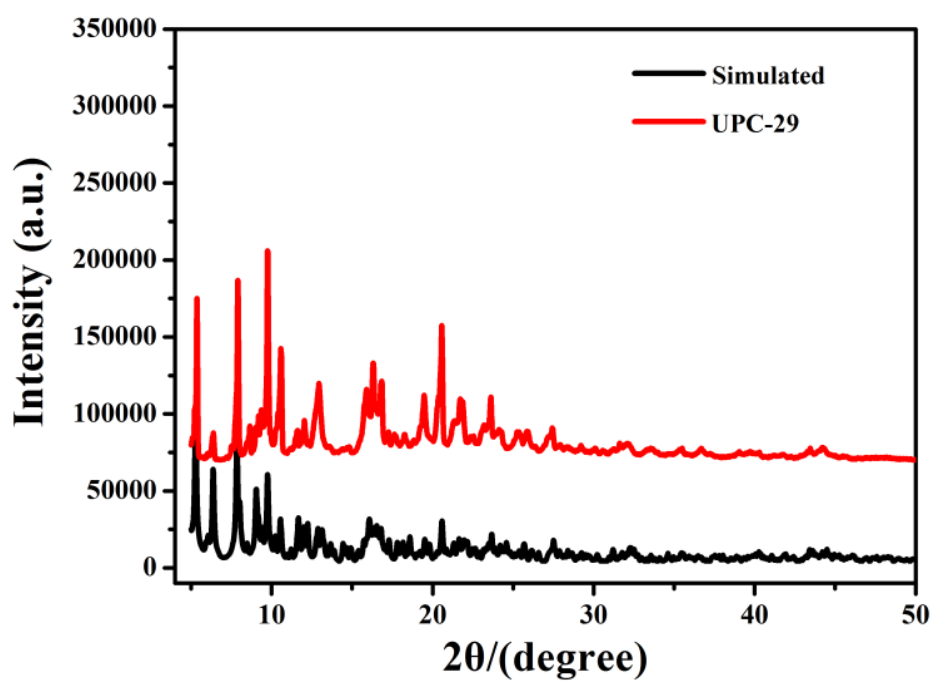

Figure S3. The PXRD patterns of UPC-29.

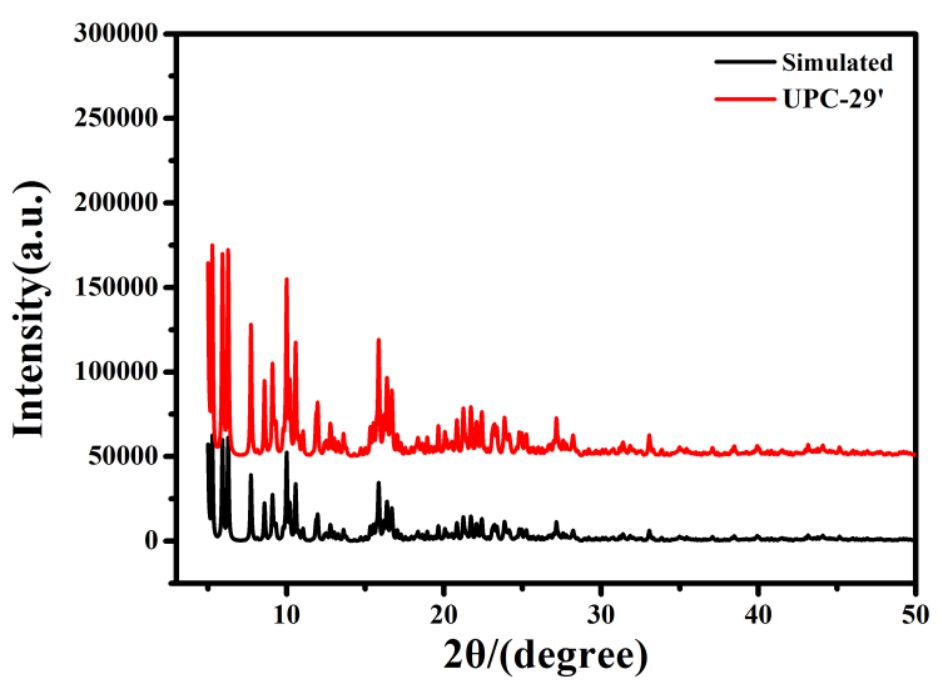

Figure S4. The PXRD patterns of UPC-29'.

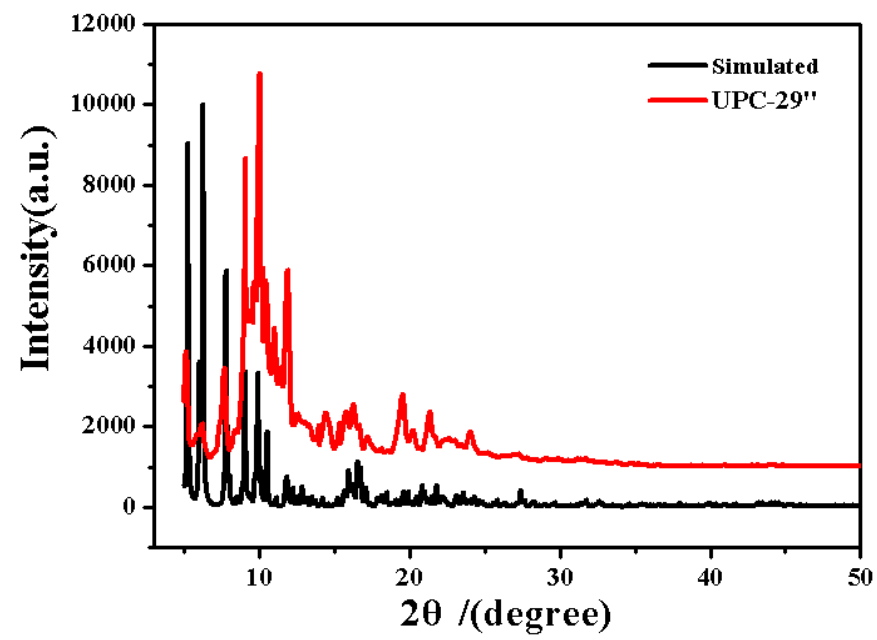

Figure S5. The PXRD patterns of UPC-29". 


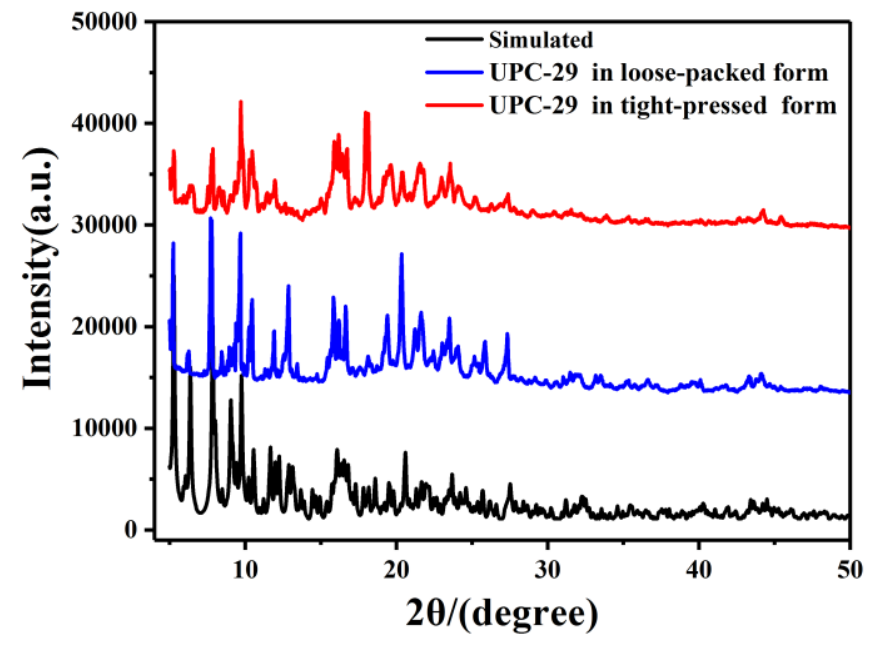

Figure S6. The PXRD patterns of UPC-29 after oil/water separation in different form.

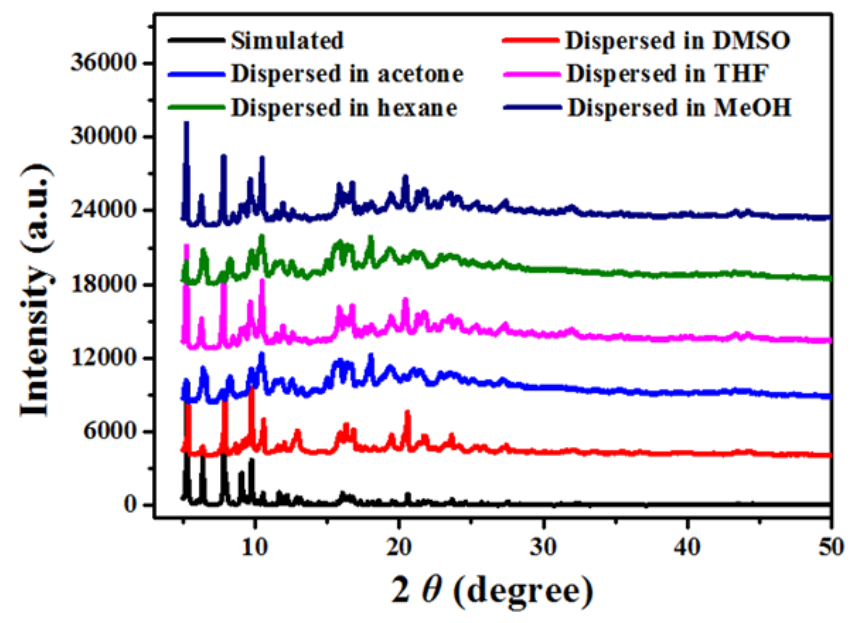

Figure. S7: The PXRD patterns of UPC-29 in different solvents

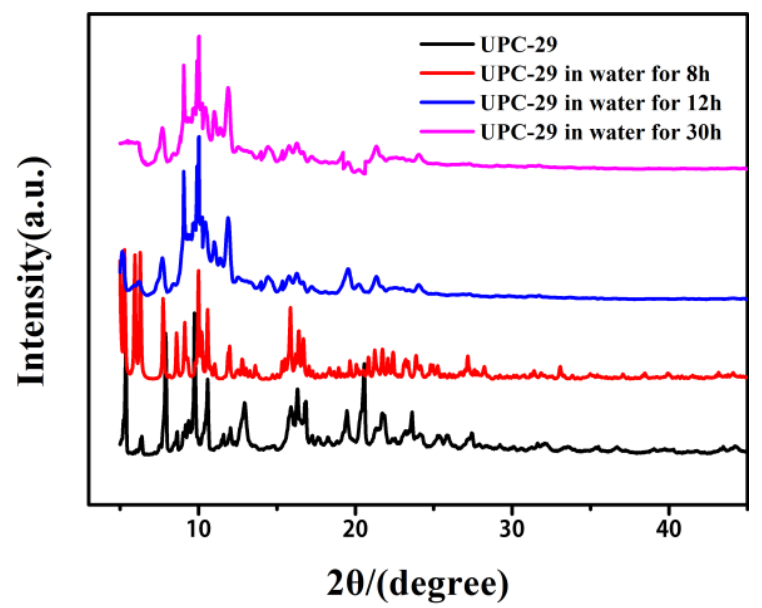

Figure. S8: The PXRD patterns of UPC-29 immersed in water for different time. 
Note: Powder X-ray diffraction data indicate that UPC-29 keep its framework intact after being immersed in water for 30 hours, even if UPC-29 has transferred to UPC-29"' in a single-crystal-to-single-crystal fashion.

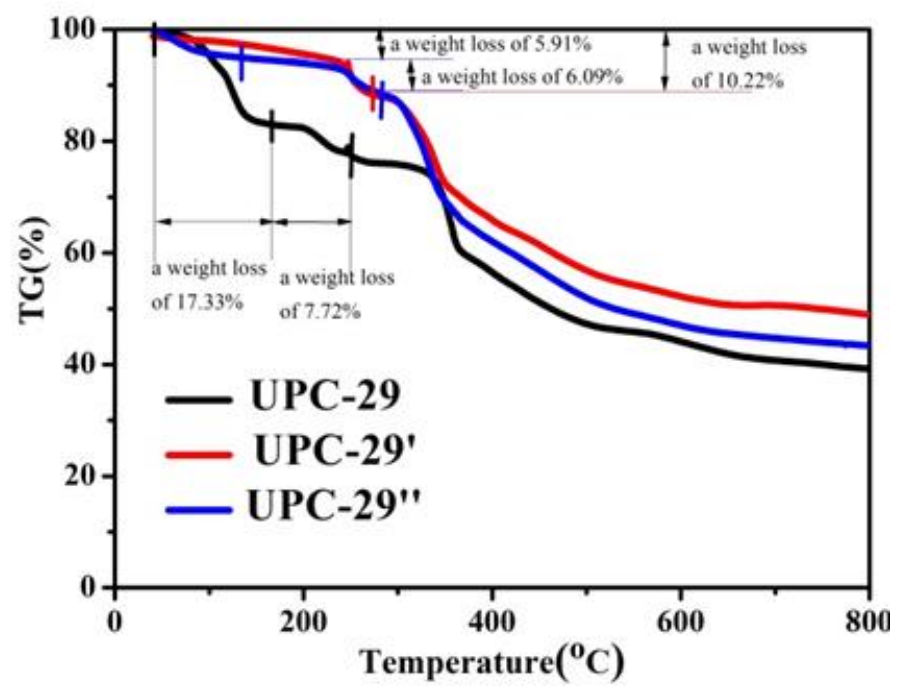

Figure S9. TGA curves for UPC-29.

The TG analyses were performed under a nitrogen atmosphere $\left(100 \mathrm{~mL} \mathrm{~min}^{-1}\right)$ from 25 to $800{ }^{\circ} \mathrm{C}$ on the polycrystalline samples of UPC-29. The TG curves are shown in Fig. S8. For UPC-29, the first step weight loss is $17.33 \%$ before $165^{\circ} \mathrm{C}$, which corresponds to the loss of 5 uncoordinated DMF molecules (calculated as $17.43 \%$ ). While the weight loss of $7.72 \%$ during the second step between 188 and $287{ }^{\circ} \mathrm{C}$ is ascribed to the loss of 2 coordinated DMF molecules (calculated as $6.97 \%$ ). Beyond $316{ }^{\circ} \mathrm{C}$, UPC-29 starts to decompose. For UPC-29', in the range of 40 to $280{ }^{\circ} \mathrm{C}$, the weight loss is $10.22 \%$ corresponding to the loss of $2 \mathrm{H}_{2} \mathrm{O}$ and $2 \mathrm{DMF}$ molecules (calculated as $10.31 \%$ ). Then the host framework begins to collapse at $285^{\circ} \mathrm{C}$. For UPC-29', the first weight loss is $5.91 \%$ in the temperature of $159{ }^{\circ} \mathrm{C}$, which is consistent with the removal of 6 uncoordinated $\mathrm{H}_{2} \mathrm{O}$ molecules (calculated as $6.00 \%$ ). The second weight loss is $6.09 \%$ in the range of $206-272{ }^{\circ} \mathrm{C}$, which could be attributed to the release of one uncoordinated DMF molecule and two coordinated $\mathrm{H}_{2} \mathrm{O}$ molecules (calculated as $6.06 \%$ ). Above $288{ }^{\circ} \mathrm{C}$, the compound begins to decompose.

The flux follows the formula below ${ }^{1-3}$ :

$$
J_{\mathrm{v}}=\frac{V}{A \Delta t}
$$


where $V$ is the volume of permeate oil or water $(\mathrm{L}), A$ is the effective area of the samples for oil/ water separation $\left(\mathrm{m}^{2}\right)$, and $\Delta \mathrm{t}$ is the permeation time $(\mathrm{h})$. The calculated results are shown in the following table:

Table S1: The calculated flux

\begin{tabular}{lll}
\hline & In the loose-packed form & In the tight - pressed form \\
\hline naphtha/water & 471.81 & 707.71 \\
gasoline/water & 294.88 & 505.51 \\
diesel/water & 337.01 & 561.67 \\
\hline
\end{tabular}




\section{Crystal Data}

Table S2: Crystal Data for UPC-29, UPC-29' and UPC-29',

\begin{tabular}{|c|c|c|c|}
\hline Compound & UPC-29 & UPC-29' & UPC-29"' \\
\hline Empirical formula & $\mathrm{C}_{121} \mathrm{H}_{113} \mathrm{Cu}_{2} \mathrm{~N}_{7} \mathrm{O}_{19}$ & $\mathrm{C}_{115} \mathrm{H}_{121} \mathrm{Cu}_{2} \mathrm{~N}_{5} \mathrm{O}_{28}$ & $\mathrm{C}_{103} \mathrm{H}_{87} \mathrm{Cu}_{2} \mathrm{NO}_{21}$ \\
\hline Formula weight & 2069.33 & 2148.24 & 1801.8 \\
\hline Crystal system & triclinic & triclinic & triclinic \\
\hline Space group & $\mathrm{P}-1$ & $\mathrm{P}-1$ & $\mathrm{P}-1$ \\
\hline$a / \AA$ & 16. $4904(5)$ & $16.7612(4)$ & $16.6272(8)$ \\
\hline$b / \AA$ & $19.7170(7)$ & $19.8555(4)$ & $20.0062(9)$ \\
\hline$c / \AA$ & $21.0351(8)$ & $20.8675(4)$ & $20.7656(10)$ \\
\hline$\alpha /^{\circ}$ & $111.002(3)$ & $110.8052(19)$ & $110.938(4)$ \\
\hline$\beta 1^{\circ}$ & $106.005(3)$ & $108.166(2)$ & $106.381(4)$ \\
\hline$\gamma /^{\circ}$ & $101.974(3)$ & $102.025(2)$ & $102.628(4)$ \\
\hline Volume $/ \AA^{3}$ & $5769.0(4)$ & $5756.6(2)$ & $5783.0(5)$ \\
\hline$Z$ & 2 & 2 & 2 \\
\hline$\rho_{\text {calc } \mathrm{mg} / \mathrm{mm}^{3}}$ & 0.996 & 0.967 & 0.931 \\
\hline$\square \mu / \mathrm{mm}^{-1}$ & 0.871 & 0.857 & 0.838 \\
\hline$F(000)$ & 1796.0 & 1736.0 & 1676.0 \\
\hline Reflections collected & 40545 & 41076 & 40263 \\
\hline Independent reflections & $\begin{array}{l}20308\left[\mathrm{R}_{\mathrm{int}}=0.0312,\right. \\
\left.\mathrm{R}_{\text {sigma }}=0.0469\right]\end{array}$ & $\begin{array}{l}20273\left[\mathrm{R}_{\mathrm{int}}=0.0209,\right. \\
\left.\mathrm{R}_{\text {sigma }}=0.0287\right]\end{array}$ & $\begin{array}{l}20387\left[R_{\text {int }}=0.0494,\right. \\
\left.R_{\text {sigma }}=0.0929\right]\end{array}$ \\
\hline Data/restraints/parameters & $20308 / 0 / 1122$ & $20273 / 0 / 1084$ & $20387 / 0 / 1045$ \\
\hline Goodness-of-fit on $F^{2}$ & 1.055 & 1.070 & 1.053 \\
\hline Final $R$ indexes $[I>=2 \sigma(\mathrm{I})]$ & $R_{1}=0.0516$ & $R_{1}=0.0459$ & $R_{1}=0.0631$ \\
\hline Final $R$ indexes [all data] & $R_{1}=0.0608$ & $R_{1}=0.0514$ & $R_{1}=0.1019$ \\
\hline Largest diff. peak/hole/e $\AA^{-3}$ & $0.76 /-0.70$ & $0.76 /-0.54$ & $1.29 /-0.76$ \\
\hline
\end{tabular}


Table S3. Selected bond length $(\AA)$ and angles $\left({ }^{\circ}\right)$ for UPC-29.

\begin{tabular}{|c|c|c|c|}
\hline $\mathrm{Cu} 1-\mathrm{Cu} 2$ & $2.6181(5)$ & $\mathrm{Cu} 2-\mathrm{O} 1$ & $1.9630(18)$ \\
\hline $\mathrm{Cu} 1-\mathrm{O} 5$ & $1.9688(18)$ & $\mathrm{Cu} 2-\mathrm{O} 7$ & $1.9663(18)$ \\
\hline $\mathrm{Cu} 1-\mathrm{O} 2$ & $1.9612(18)$ & $\mathrm{Cu} 2-\mathrm{O} 8$ & $1.9890(19)$ \\
\hline $\mathrm{Cu} 1-\mathrm{O} 4$ & 1.9537 (19) & $\mathrm{Cu} 2-\mathrm{O} 3$ & 1.9855 (19) \\
\hline $\mathrm{Cu} 1-\mathrm{O} 6$ & $1.9536(19)$ & $\mathrm{Cu} 2-\mathrm{O} 9$ & $2.160(2)$ \\
\hline $\mathrm{Cu} 1-\mathrm{O} 10$ & $2.162(2)$ & & \\
\hline $\mathrm{O} 5-\mathrm{Cu} 1-\mathrm{Cu} 2$ & $82.68(6)$ & $\mathrm{O} 1-\mathrm{Cu} 2-\mathrm{Cu} 1$ & $82.82(6)$ \\
\hline $\mathrm{O} 5-\mathrm{Cu} 1-\mathrm{O} 10$ & $99.20(8)$ & $\mathrm{O} 1-\mathrm{Cu} 2-\mathrm{O} 7$ & $167.62(8)$ \\
\hline $\mathrm{O} 2-\mathrm{Cu} 1-\mathrm{Cu} 2$ & $84.96(6)$ & $\mathrm{O} 1-\mathrm{Cu} 2-\mathrm{O} 8$ & $92.69(8)$ \\
\hline $\mathrm{O} 2-\mathrm{Cu} 1-\mathrm{O} 5$ & $167.58(8)$ & $\mathrm{O} 1-\mathrm{Cu} 2-\mathrm{O} 3$ & $89.36(8)$ \\
\hline $\mathrm{O} 2-\mathrm{Cu} 1-\mathrm{O} 10$ & $93.02(8)$ & $\mathrm{O} 1-\mathrm{Cu} 2-\mathrm{O} 9$ & $91.60(8)$ \\
\hline $\mathrm{O} 4-\mathrm{Cu} 1-\mathrm{Cu} 2$ & $84.28(6)$ & $\mathrm{O} 7-\mathrm{Cu} 2-\mathrm{Cu} 1$ & $84.83(6)$ \\
\hline $\mathrm{O} 4-\mathrm{Cu} 1-\mathrm{O} 5$ & $90.24(8)$ & $\mathrm{O} 7-\mathrm{Cu} 2-\mathrm{O} 8$ & $86.81(8)$ \\
\hline $\mathrm{O} 4-\mathrm{Cu} 1-\mathrm{O} 2$ & $89.79(8)$ & $\mathrm{O} 7-\mathrm{Cu} 2-\mathrm{O} 3$ & $88.45(8)$ \\
\hline $\mathrm{O} 4-\mathrm{Cu} 1-\mathrm{O} 6$ & $167.55(8)$ & $\mathrm{O} 7-\mathrm{Cu} 2-\mathrm{O} 9$ & $100.73(8)$ \\
\hline $\mathrm{O} 4-\mathrm{Cu} 1-\mathrm{O} 10$ & $100.00(8)$ & $\mathrm{O} 8-\mathrm{Cu} 2-\mathrm{Cu} 1$ & $84.03(6)$ \\
\hline $\mathrm{O} 6-\mathrm{Cu} 1-\mathrm{Cu} 2$ & $83.40(6)$ & $\mathrm{O} 8-\mathrm{Cu} 2-\mathrm{O} 9$ & $97.74(8)$ \\
\hline $\mathrm{O} 6-\mathrm{Cu} 1-\mathrm{O} 5$ & $86.42(8)$ & $\mathrm{O} 3-\mathrm{Cu} 2-\mathrm{Cu} 1$ & $83.51(6)$ \\
\hline $\mathrm{O} 6-\mathrm{Cu} 1-\mathrm{O} 2$ & $90.91(8)$ & $\mathrm{O} 3-\mathrm{Cu} 2-\mathrm{O} 8$ & $167.02(8)$ \\
\hline $\mathrm{O} 6-\mathrm{Cu} 1-\mathrm{O} 10$ & $92.38(8)$ & $\mathrm{O} 3-\mathrm{Cu} 2-\mathrm{O} 9$ & $95.01(8)$ \\
\hline $\mathrm{O} 10-\mathrm{Cu} 1-\mathrm{Cu} 2$ & $175.29(6)$ & $\mathrm{O} 9-\mathrm{Cu} 2-\mathrm{Cu} 1$ & $174.23(6)$ \\
\hline
\end{tabular}


Table S4. Selected bond length $(\AA)$ and angles $\left({ }^{\circ}\right)$ for UPC-29'.

\begin{tabular}{|c|c|c|c|}
\hline $\mathrm{Cu} 1-\mathrm{Cu} 3$ & $2.6348(5)$ & $\mathrm{Cu} 3-\mathrm{O} 1$ & $1.9864(17)$ \\
\hline $\mathrm{Cu} 1-\mathrm{O} 4$ & $1.9829(15)$ & $\mathrm{Cu} 3-\mathrm{O} 6$ & $1.9728(16)$ \\
\hline $\mathrm{Cu} 1-\mathrm{O} 8$ & $1.9451(16)$ & $\mathrm{Cu} 3-\mathrm{O} 10$ & $1.9766(16)$ \\
\hline $\mathrm{Cu} 1-\mathrm{O} 11$ & $1.9546(16)$ & $\mathrm{Cu} 3-\mathrm{O} 19$ & $1.9590(16)$ \\
\hline $\mathrm{Cu} 1-\mathrm{O} 13$ & $1.9870(15)$ & $\mathrm{Cu} 3-\mathrm{O} 27$ & $2.133(2)$ \\
\hline $\mathrm{Cu} 1-\mathrm{O} 25$ & $2.1501(17)$ & & \\
\hline $\mathrm{O} 4-\mathrm{Cu} 1-\mathrm{Cu} 3$ & $81.53(5)$ & $\mathrm{O} 1-\mathrm{Cu} 3-\mathrm{Cu} 1$ & $82.95(5)$ \\
\hline $\mathrm{O} 4-\mathrm{Cu} 1-\mathrm{O} 13$ & $166.83(7)$ & $\mathrm{O} 1-\mathrm{Cu} 3-\mathrm{O} 27$ & 95.77 (10) \\
\hline $\mathrm{O} 4-\mathrm{Cu} 1-\mathrm{O} 25$ & $99.83(7)$ & $\mathrm{O} 6-\mathrm{Cu} 3-\mathrm{Cu} 1$ & $81.99(5)$ \\
\hline $\mathrm{O} 8-\mathrm{Cu} 1-\mathrm{Cu} 3$ & $84.06(5)$ & $\mathrm{O} 6-\mathrm{Cu} 3-\mathrm{O} 1$ & $88.71(7)$ \\
\hline $\mathrm{O} 8-\mathrm{Cu} 1-\mathrm{O} 4$ & $90.09(7)$ & $\mathrm{O} 6-\mathrm{Cu} 3-\mathrm{O} 10$ & $167.98(7)$ \\
\hline $\mathrm{O} 8-\mathrm{Cu} 1-\mathrm{O} 11$ & $168.47(7)$ & $\mathrm{O} 6-\mathrm{Cu} 3-\mathrm{O} 27$ & $92.53(9)$ \\
\hline $\mathrm{O} 8-\mathrm{Cu} 1-\mathrm{O} 13$ & $85.59(7)$ & $\mathrm{O} 10-\mathrm{Cu} 3-\mathrm{Cu} 1$ & $86.00(5)$ \\
\hline $\mathrm{O} 8-\mathrm{Cu} 1-\mathrm{O} 25$ & $99.39(7)$ & $\mathrm{O} 10-\mathrm{Cu} 3-\mathrm{O} 1$ & $89.25(7)$ \\
\hline $\mathrm{O} 11-\mathrm{Cu} 1-\mathrm{Cu} 3$ & $84.54(5)$ & $\mathrm{O} 10-\mathrm{Cu} 3-\mathrm{O} 27$ & $99.46(9)$ \\
\hline $\mathrm{O} 11-\mathrm{Cu} 1-\mathrm{O} 4$ & $89.98(7)$ & $\mathrm{O} 19-\mathrm{Cu} 3-\mathrm{Cu} 1$ & $83.20(5)$ \\
\hline $\mathrm{O} 11-\mathrm{Cu} 1-\mathrm{O} 13$ & $91.79(7)$ & $\mathrm{O} 19-\mathrm{Cu} 3-\mathrm{O} 1$ & $166.00(7)$ \\
\hline $\mathrm{O} 11-\mathrm{Cu} 1-\mathrm{O} 25$ & $91.96(7)$ & $\mathrm{O} 19-\mathrm{Cu} 3-\mathrm{O} 6$ & $91.36(7)$ \\
\hline $\mathrm{O} 13-\mathrm{Cu} 1-\mathrm{Cu} 3$ & $85.65(5)$ & $\mathrm{O} 19-\mathrm{Cu} 3-\mathrm{O} 10$ & $87.79(7)$ \\
\hline $\mathrm{O} 13-\mathrm{Cu} 1-\mathrm{O} 25$ & $93.15(7)$ & $\mathrm{O} 19-\mathrm{Cu} 3-\mathrm{O} 27$ & $98.21(10)$ \\
\hline $\mathrm{O} 25-\mathrm{Cu} 1-\mathrm{Cu} 3$ & $176.26(5)$ & $\mathrm{O} 27-\mathrm{Cu} 3-\mathrm{Cu} 1$ & $174.39(7)$ \\
\hline
\end{tabular}


Table S5. Selected bond length $(\AA)$ and angles $\left({ }^{\circ}\right)$ for UPC-29".

\begin{tabular}{|c|c|c|c|}
\hline $\mathrm{Cu} 1-\mathrm{Cu} 2$ & 2.6147 (7) & $\mathrm{Cu} 2-\mathrm{O} 2^{\mathrm{i}}$ & $1.973(2)$ \\
\hline $\mathrm{Cu} 1-\mathrm{O} 1$ & $1.944(2)$ & $\mathrm{Cu} 2-\mathrm{O} 3$ & $1.947(2)$ \\
\hline $\mathrm{Cu} 1-\mathrm{O} 4$ & $1.971(3)$ & $\mathrm{Cu} 2-\mathrm{O} 5$ & $1.966(2)$ \\
\hline $\mathrm{Cu} 1-\mathrm{O} 6$ & $1.943(2)$ & $\mathrm{Cu} 2-\mathrm{O} 9$ & $1.956(3)$ \\
\hline $\mathrm{Cu} 1-\mathrm{O} 8$ & $1.958(2)$ & $\mathrm{Cu} 2-\mathrm{O} 14$ & $2.126(3)$ \\
\hline $\mathrm{Cu} 1-\mathrm{O} 13$ & $2.154(3)$ & $\mathrm{O} 2-\mathrm{Cu} 2^{\mathrm{ii}}$ & $1.973(2)$ \\
\hline $\mathrm{O} 1-\mathrm{Cu} 1-\mathrm{Cu} 2$ & $84.41(8)$ & $\mathrm{O} 2-\mathrm{Cu} 2-\mathrm{O} 14$ & $97.76(11)$ \\
\hline $\mathrm{O} 1-\mathrm{Cu} 1-\mathrm{O} 4$ & $90.41(10)$ & $\mathrm{O} 3-\mathrm{Cu} 2-\mathrm{Cu} 1$ & $83.47(8)$ \\
\hline $\mathrm{O} 1-\mathrm{Cu} 1-\mathrm{O} 8$ & 85.69 (10) & $\mathrm{O} 3-\mathrm{Cu} 2-\mathrm{O} 2^{\mathrm{i}}$ & $166.63(11)$ \\
\hline $\mathrm{O} 1-\mathrm{Cu} 1-\mathrm{O} 13$ & $100.11(10)$ & $\mathrm{O} 3-\mathrm{Cu} 2-\mathrm{O} 5$ & $92.13(11)$ \\
\hline $\mathrm{O} 4-\mathrm{Cu} 1-\mathrm{Cu} 2$ & $83.10(8)$ & $\mathrm{O} 3-\mathrm{Cu} 2-\mathrm{O} 9$ & $88.56(11)$ \\
\hline $\mathrm{O} 4-\mathrm{Cu} 1-\mathrm{O} 13$ & $98.70(11)$ & $\mathrm{O} 3-\mathrm{Cu} 2-\mathrm{O} 14$ & $95.60(11)$ \\
\hline $\mathrm{O} 6-\mathrm{Cu} 1-\mathrm{Cu} 2$ & $84.91(8)$ & $\mathrm{O} 5-\mathrm{Cu} 2-\mathrm{Cu} 1$ & $83.70(8)$ \\
\hline $\mathrm{O} 6-\mathrm{Cu} 1-\mathrm{O} 1$ & $169.17(11)$ & $\mathrm{O} 5-\mathrm{Cu} 2-\mathrm{O} 2^{\mathrm{i}}$ & $87.38(10)$ \\
\hline $\mathrm{O} 6-\mathrm{Cu} 1-\mathrm{O} 4$ & 90.07 (10) & $\mathrm{O} 5-\mathrm{Cu} 2-\mathrm{O} 14$ & $94.11(11)$ \\
\hline $\mathrm{O} 6-\mathrm{Cu} 1-\mathrm{O} 8$ & $91.54(11)$ & $\mathrm{O} 9-\mathrm{Cu} 2-\mathrm{Cu} 1$ & $84.90(8)$ \\
\hline $\mathrm{O} 6-\mathrm{Cu} 1-\mathrm{O} 13$ & $90.51(10)$ & $\mathrm{O} 9-\mathrm{Cu} 2-\mathrm{O} 2^{\mathrm{i}}$ & $89.29(11)$ \\
\hline $\mathrm{O} 8-\mathrm{Cu} 1-\mathrm{Cu} 2$ & $84.63(8)$ & $\mathrm{O} 9-\mathrm{Cu} 2-\mathrm{O} 5$ & $168.43(11)$ \\
\hline $\mathrm{O} 8-\mathrm{Cu} 1-\mathrm{O} 4$ & $167.43(11)$ & $\mathrm{O} 9-\mathrm{Cu} 2-\mathrm{O} 14$ & $97.32(12)$ \\
\hline $\mathrm{O} 8-\mathrm{Cu} 1-\mathrm{O} 13$ & $93.76(10)$ & $\mathrm{O} 14-\mathrm{Cu} 2-\mathrm{Cu} 1$ & $177.58(8)$ \\
\hline $\mathrm{O} 13-\mathrm{Cu} 1-\mathrm{Cu} 2$ & $175.09(7)$ & $\mathrm{O} 2-\mathrm{Cu} 2-\mathrm{O} 14$ & $97.76(11)$ \\
\hline $\mathrm{O} 2^{\mathrm{i}}-\mathrm{Cu} 2-\mathrm{Cu} 1$ & $83.19(7)$ & $\mathrm{O} 3-\mathrm{Cu} 2-\mathrm{Cu} 1$ & $83.47(8)$ \\
\hline
\end{tabular}




\section{Photographs and SEM images}

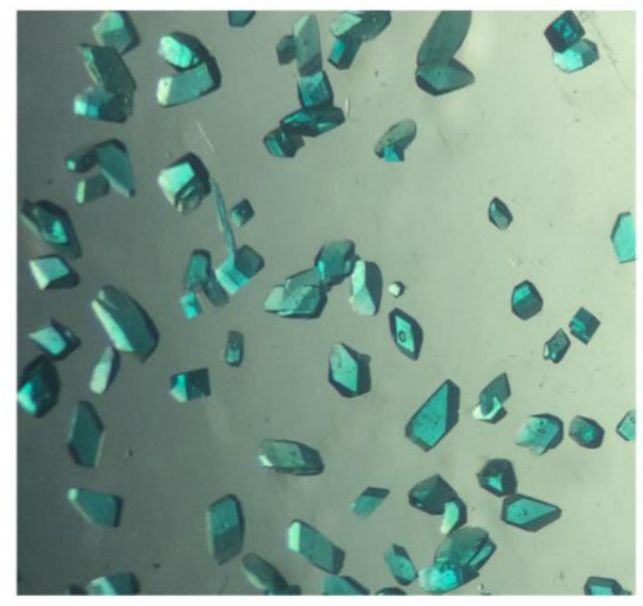

Figure S10. Photograph of crystals UPC-29.

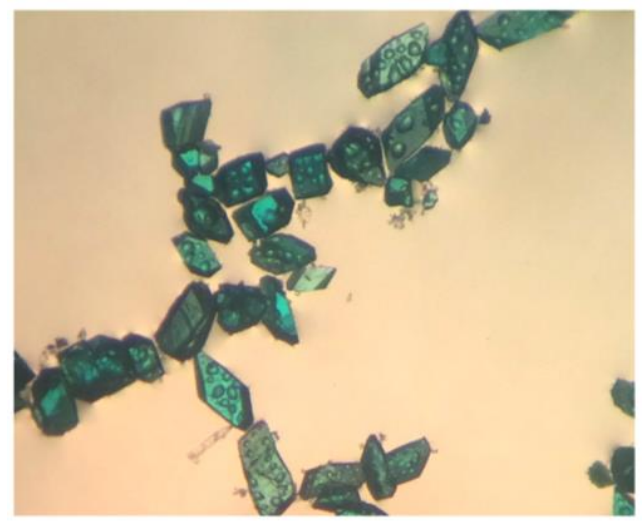

Figure S11. Photograph of crystals UPC-29 soaked in water.

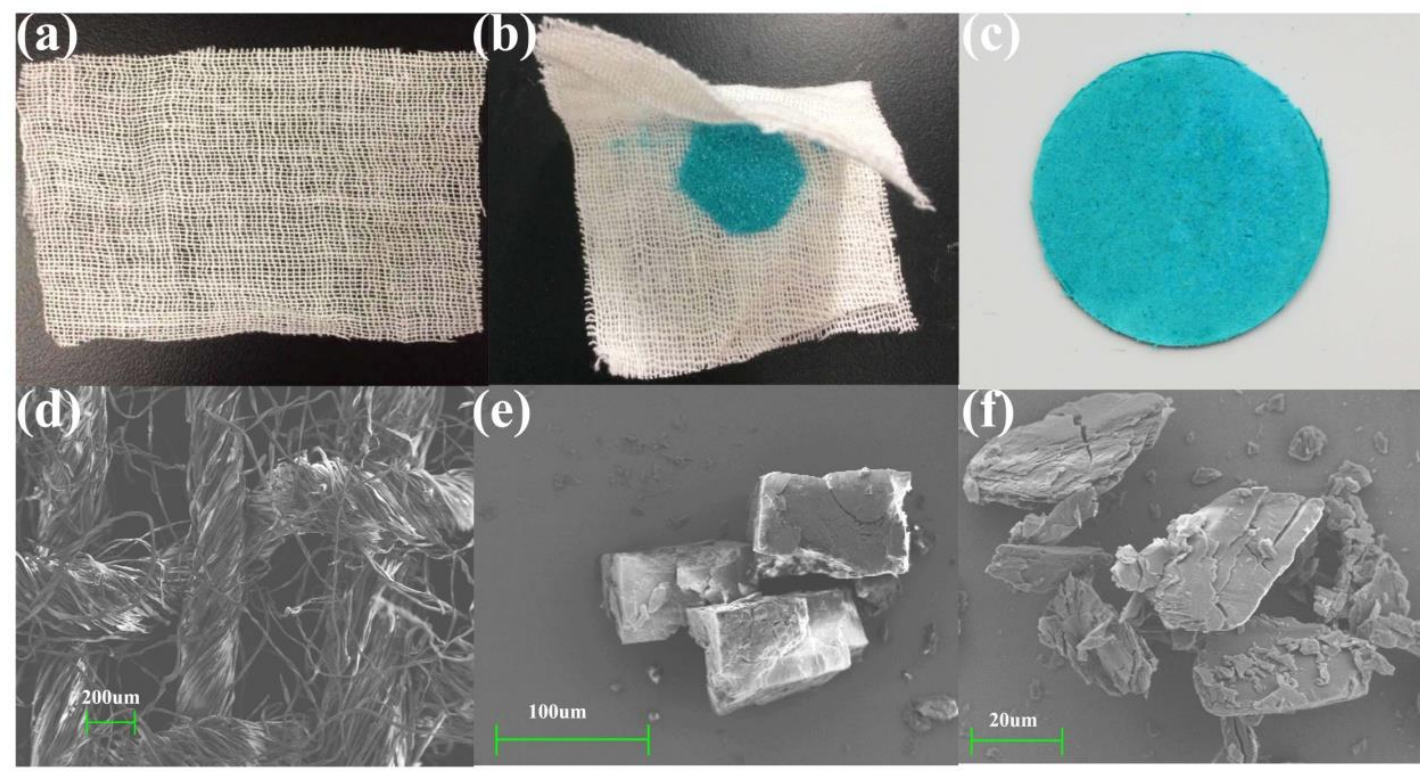

Figure S12. (a) Photograph of gauze; (b) The state of crystals packaged by gauze; (c) Photograph of tablet-shaped samples; SEM images of gauze (d), crystals (e) and ground crystals (f). 


\section{Water sorption isotherms}

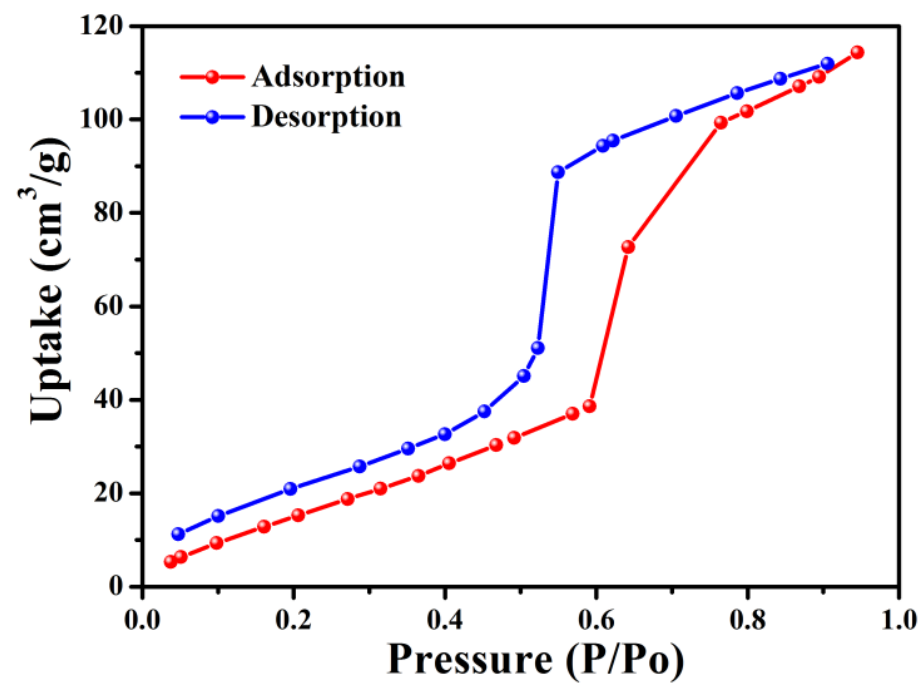

Fig. S13. Water sorption isotherms for UPC-29 at $298 \mathrm{~K}$

In order to explore the interaction between water and UPC-29, the water vapor adsorption isotherm was measured at $298 \mathrm{~K}$. According to IUPAC, The water sorption isotherms are divided into seven types to classify the hydrophilicity or hydrophobicity of the sorbent. ${ }^{4}$ As depicted in Fig. S13, UPC-29 exhibits a type V water sorption isotherm, categorized as a hydrophobic or weakly hydrophilic material, which reveals that UPC-29 adsorbs small amount of water at $P / P_{0}<0.6$ until the sorption capacity increases suddenly. 
10 Water contact angle for UPC-29 and other MOFs

Table S6: Water contact angle for UPC-29 and other MOFs.

\begin{tabular}{|c|c|c|c|}
\hline MOFs & Surface modification Type & Contact angle $\left[{ }^{\circ}\right]$ & Ref. \\
\hline PESD-1 & aromatic ring & $>150$ & 5 \\
\hline MOFF-1 & Fluorinated aromatic ring & $108 \pm 2$ & 6 \\
\hline MOFF-2 & Fluorinated aromatic ring & $151 \pm 1$ & 6 \\
\hline MOFF-3 & Fluorinated aromatic ring & $135 \pm 2$ & 6 \\
\hline IRMOF-3-AM4 & alkyl chain $\left(\mathrm{C}_{4}\right)$ & $116 \pm 6$ & 7 \\
\hline IRMOF-3-AM5 & alkyl chain $\left(\mathrm{C}_{5}\right)$ & $119 \pm 10$ & 7 \\
\hline IRMOF-3-AM6 & alkyl chain $\left(\mathrm{C}_{6}\right)$ & $124 \pm 8$ & 7 \\
\hline IRMOF-3-AM15 & alkyl chain $\left(\mathrm{C}_{15}\right)$ & $123 \pm 5$ & 7 \\
\hline IRMOF-3-AMiPr & alkyl chain & $125 \pm 12$ & 7 \\
\hline IRMOF-3-AMiBu & alkyl chain & $105 \pm 11$ & 7 \\
\hline MIL-53(Al)-AM4 & alkyl chain $\left(\mathrm{C}_{4}\right)$ & $>150$ & 7 \\
\hline MIL-53(Al)-AM6 & alkyl chain $\left(\mathrm{C}_{6}\right)$ & $>150$ & 7 \\
\hline HFGO@ZIF-8 & $\begin{array}{l}\text { highly fluorinated } \\
\text { graphene oxide }\end{array}$ & 162 & 8 \\
\hline PDMS-MOF-5 & Polydimethysiloxane coating & 128 & 9 \\
\hline PDMS-HKUST-1 & polydimethysiloxane coating & 130 & 9 \\
\hline PDMS-ZnBT & polydimethysiloxane coating & 130 & 9 \\
\hline pbdc-9a & alkyl chain $\left(\mathrm{C}_{9}\right)$ & $113 \pm 2$ & 10 \\
\hline pbdc-10a & alkyl chain $\left(\mathrm{C}_{10}\right)$ & $110 \pm 1$ & 10 \\
\hline pbdc-11a & alkyl chain $\left(C_{11}\right)$ & $114 \pm 1$ & 10 \\
\hline pbdc-12a & alkyl chain $\left(\mathrm{C}_{12}\right)$ & $120 \pm 2$ & 10 \\
\hline
\end{tabular}


UPC-21

functionalized aromatic

NH2-MIL-125(Ti)

No

$16 \pm 2$

ZIF-67

DC 1-2577

$16 \pm 2$

SH NH2-MIL-125

varying-length aliphatic carbon

$146 \pm 3$

14

SH ZIF-67

$\mathrm{Cu}_{3}\left(\mathrm{NH}_{2} \mathrm{BTC}\right)_{2}$ chains

$147 \pm 6$

OPA-UiO-66

n-octadecylphosphonic acid

160

16

ZIF-7

$\mathrm{ZnO}$ array coatings

151.3

17

ZIF-7-300

octadecylamine

154.7

MIL-101(Cr)

$156 \pm 1$

18

UiO-66

monoamines of various alkyl chain lengths

$154 \pm 1$

$134 \pm 0.69$

19

UPC-29

in loose-packed form

$178 \pm 1$

This work 


\section{Photographs of oil/water separation}
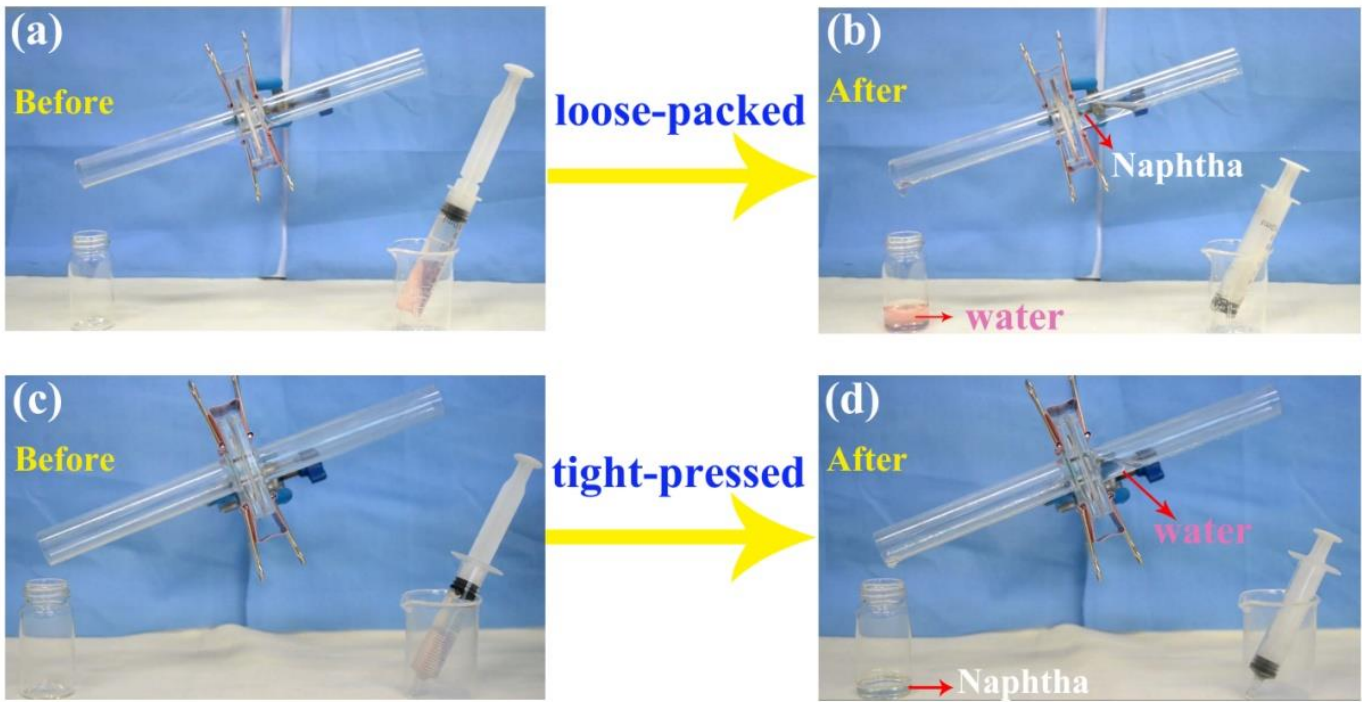

Figure S14. Photographs of naphtha/water separation experiments: Initial (a) and final states (b) of the separation experiments in the loose-packed form and initial (c) and final states (d) of the separation experiments in the tight-pressed form.
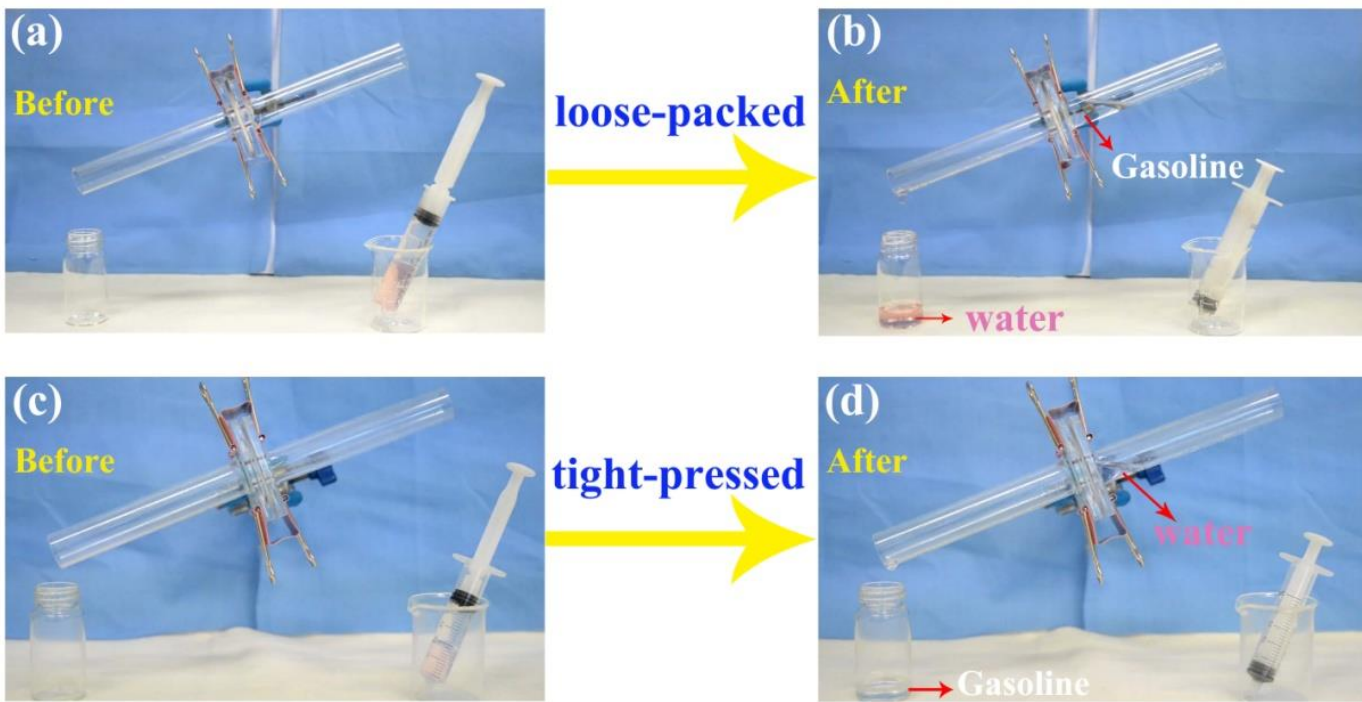

Figure S15. Photographs of gasoline/water separation experiments: Initial (a) and final states (b) of the separation experiments in the loose-packed form and initial (c) and final states (d) of the separation experiments in the tight-pressed form. 


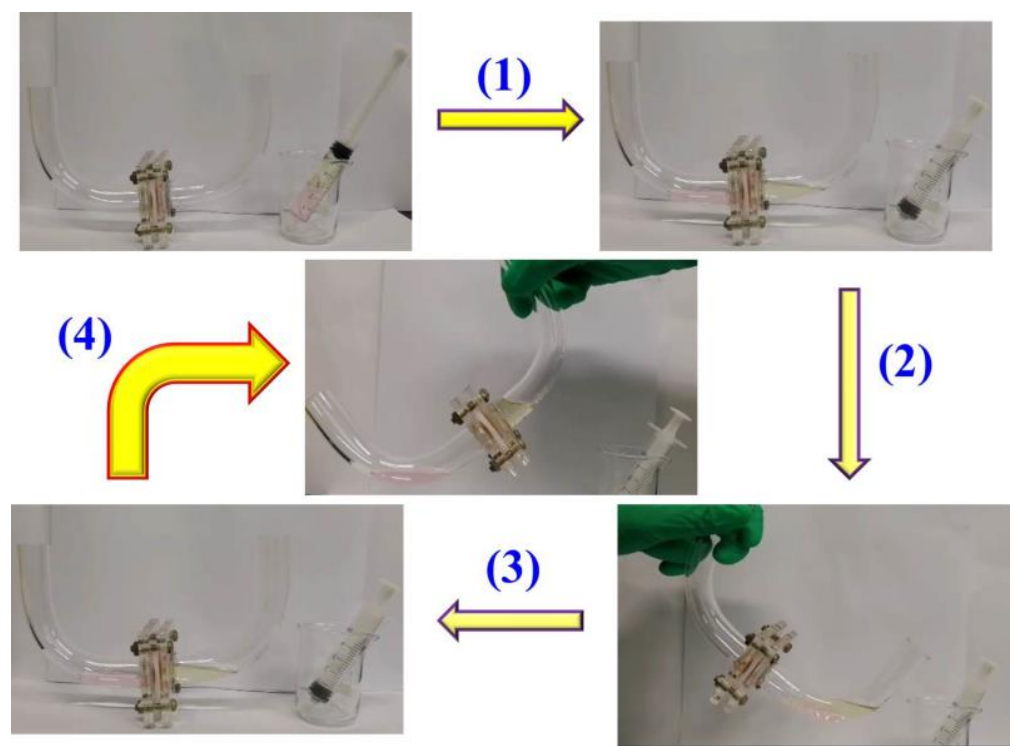

Figure S16. The oil/water separation photograph of crystals UPC-29 in U-tube filter. 


\section{References}

1. Li, J.; Xu, C. C.; Guo, C. H.; Tian, H. F.; Zha, F.; Guo, L. Underoil superhydrophilic desert sand layer for efficient gravity-directed water-in-oil emulsions separation with high flux. $J$. Mater. Chem. A, 2018, 6, 223-230.

2. Wei, J.; Qiu, C. Q.; Tang, C. Y.; Wang, R.; Fane, A. G. Synthesis and characterization of flatsheet thin film composite forward osmosis membranes. J. MemSci., 2011, 372, 292-302.

3. Wang, J.; Wang, Y. M.; Zhang, Y. T.; Uliana, A.; Zhu, J. Y.; Liu, J. D;. Bruggen, B. V. Zeolitic Imidazolate Framework/Graphene Oxide Hybrid Nanosheets Functionalized Thin Film Nanocomposite Membrane for Enhanced Antimicrobial Performance. ACS Appl. Mater. Interfaces, 2016, 8, 25508-25519.

4. Ng, E. P.; Mintova, S. Nanoporous materials with enhanced hydrophilicity and high water sorption capacity Mintova, Microporous Mesoporous Mater., 2008, 114, 1-26.

5. Rao, K. P.; Higuchi, M.; Sumida, K.; Furukawa, S.; Duan, J. G.; Kitagawa, S. Design of Superhydrophobic Porous Coordination Polymers through the Introduction of External Surface Corrugation by the Use of an Aromatic Hydrocarbon Building Unit Angew. Chem. Int. $E d .$, 2014, 53, 8225-8230.

6. Chen, T. H.; Popov, I.; Zenasni, O.; Daugulis, O.; Miljanic, O.; Superhydrophobic perfluorinated metal-organic frameworks Chem. Commun. 2013, 49, 6846-6848.

7. Nguyen, J. G.; Cohen, S. M. Moisture-Resistant and Superhydrophobic Metal-Organic Frameworks Obtained via Postsynthetic Modification J. Am. Chem. Soc., 2010, 132, 45604561.

8. Jayaramulu, K.; Datta, K. K. R.; Rösler, C. Petr, M.; Otyepka, M.; Zboril, R.; Fischer, R. A. Biomimetic Superhydrophobic/Superoleophilic Highly Fluorinated Graphene Oxide and ZIF8 Composites for Oil-Water Separation Angew. Chem. Int. Ed., 2016, 55, 1178-1182.

9. Zhang, W.; Hu, Y. L.; Ge, J.; Jiang, H. L.; Yu, S. H. A Facile and General Coating Approach to Moisture/Water-Resistant Metal-Organic Frameworks with Intact Porosity J. Am. Chem. Soc., 2014, 136, 16978-16981.

10. Zhang, Z. J.; Nguyen, H. T. H.; Miller, S. A.; Ploskonka, A. M.; DeCoste, J. B.; DeCoste, S. M. Polymer-Metal-Organic Frameworks (polyMOFs) as Water Tolerant Materials for Selective Carbon Dioxide Separations J. Am. Chem. Soc., 2016, 138, 920-925.

11. Liu, C. Y.; Liu, Q.; Huang, A. S. A superhydrophobic zeolitic imidazolate framework (ZIF-90) with high steam stability for efficient recovery of bioalcohols Chem. Commun., 2016, 52, 3400-3402.

12. Mukherjee, S.; Kansara, A. M.; Saha, D.; Gonnade, R.; Mullangi, D.; Manna, B.; Desai, A. V.; Thorat, S. H.; Singh, P. S.; Mukherjee, A.; Ghosh, S. K. An ultra hydrophobic fluorous Metal-Organic Framework derived recyclable composite as a promising platform to tackle marine oil spills. Chem. Eur. J. 2016, 22, 10937-10943. 
13. Zhang, M. H.; Xin, X. L.; Xiao, Z. Y.; Wang, R. M.; Zhang, L. L.; Sun, D. F. A multiaromatic hydrocarbon unit induced hydrophobic metal-organic framework for efficient $\mathrm{C}_{2} / \mathrm{C}_{1}$ hydrocarbon and oil/water separation. J. Mater. Chem. A, 2017, 5, 1168-1175.

14. Qian, X. K.; Sun, F. X.; Sun, J.; Wu, H. Y.; Xiao, F. Wu, X. X.; Zhu, G. S. Imparting surface hydrophobicity to metal-organic frameworks using a facile solution-immersion process to enhance water stability for $\mathrm{CO}_{2}$ capture Nanoscale, 2017, 9, 2003-2008.

15. Rubin, H. N.; Reynolds, M. M. Functionalization of Metal-Organic Frameworks To Achieve Controllable Wettability Inorg. Chem., 2017, 56, 5266-5274.

16. Sun, Y. X.; Sun, Q.; Huang, H. L.; Aguila, B.; Niu, Z.; Perman, J. A.; Ma, S. Q. A molecularlevel superhydrophobic external surface to improve the stability of metal-organic frameworks J. Mater. Chem. A, 2017, 5, 18770-18776.

17. Zhang, G. L.; Zhang, J. H.; Su, P. C.; Xu, Z. H.; Li, W. B.; Shen, C.; Meng, Q. Non-activation MOF arrays as a coating layer to fabricate a stable superhydrophobic micro/nano flower-like architecture Chem. Commun., 2017, 53, 8340-8343.

18. Gao, M. L.; Zhao, S. Y.; Chen, Z. Y.; Liu, L.; Han, Z. B. Superhydrophobic/superoleophilic MOF composites for oil-water separation. Inorg. Chem., 2019, 58, 2261-2264.

19. Eom, S.; Kang, D. W.; Kang, M. J.; Choe, J. H.; Kim, H.; Kim, D. W.; Hong, C. S. Finetuning of wettability in a single metal-organic framework via postcoordination modification and its reduced graphene oxide aerogel for oil-water separation Chem. Sci., 2019, 10, 26632669. 